\title{
Fractinal Order Speed Control of DC Motor Using Levy Mutated Artificial Bee Colony Algorithm
}

\author{
Anguluri Rajasekhar \\ Department of Electrical and Electronics Engineering \\ National Institute of Technology-Warangal \\ Andhra Pradesh, India \\ Email: rajasekhar.anguluri@ieee.org
}

\author{
Pratap Kunathi \\ Department of Electrical and Electronics Engineering \\ National Institute of Technology-Warangal \\ Andhra Pradesh, India \\ Email: pratap.kunathi999@gmail.com
}

\author{
Ajith Abraham \\ Faculty of Computer Science and Electrical Engineering, \\ VSB - Technical University of Ostrava, Czech Republic \\ Machine Intelligence Research Labs (MIR Labs), USA \\ Email: ajith.abraham@ieee.org
}

\author{
Millie Pant \\ Department of Paper and Pulp Technology, \\ Indian Institute of Technology-Roorkee \\ Roorkee 247667, INDIA \\ Email: millifpt@iit.ernet.in
}

\begin{abstract}
In this paper we propose a new technique for designing feedback control of a DC motor speed using fractional order proportional-integral-derivative (FOPID controller). DC motor is often used in robotics and other fields of control and therefore speed control is very important. FOPID controller's parameters are composed of the proportionality constant, integral constant, derivative constant, derivative order and integral order, and its design is more complex than that of conventional integer-order proportionalintegral-derivative (PID) controllers. Here the controller synthesis is formulated as a single objective optimization problem and based on Integral Time Absolute Error (ITAE) criterion. A modified Artificial Bee Colony Algorithm is been used to tune the FOPID controller parameters.
\end{abstract}

Keywords-Artificial Bee Colony; fractional order PID controller; Levy Probability Distribution;

\section{INTRODUCTION}

DC motor is one of the oldest among all the motors that are being available today. It is a power actuator, which converts direct current electrical energy into rotational mechanical energy. DC motors are still often used in traction application, in industry and in numerous control applications, robotic manipulators, disk drive etc., as well.

In this approach we considered the armature controlled DC motor in which a constant filed current is been utilized by the motor. This kind of DC motor is been controlled by nontraditional control technique which is known as fractional-order control, a generalized version of integerorder control. Dynamic systems based on fractional order calculus $[1,2]$ have been a subject of extensive research in recent years $[3,4]$, since the proposition of concept of the fractional-order controllers and then demonstration of their effectiveness in actuating desired fractional order system responses by Podlubny in 1999 [5].

A few recent works in this direction as well as schemes for digital and hardware realizations of such systems can be figured in Chen et al [6, 7], and Nakgawa and Sorimachi [8]. Vinagre et al. [9] proposed a frequency domain approach based on expected crossover frequency and phase margin for the same controller design. Petras [10] came up with a method based on the pole distribution of the characteristic equation in the complex plane. Dorcak et al [11] proposed a state-space design approach based on feedback pole placement. The fractional controller can also be synthesized by cascading a proper fractional unit to an integer-order controller (Chengbin and Hori, [12]).

Proportional-integral-derivative (PID) controllers have been used for several decades in industries for process control applications. The reason for their wide popularity lies in the simplicity of design and good performance including low percentage overshoot and small settling time for slow process plants [13]. In fractional-order proportional-integral derivative (FOPID) controller, I and D operations are usually of fractional order; therefore, besides setting the proportional, derivative and integral constants $K_{P}, K_{d}, K_{i}$,we have two more parameters: the order of fractional integration $\mu$ and that of fractional derivative $\lambda$. Determining an optimal set of values for $K_{P}$, $K_{d}, K_{i}, \mu$ and $\lambda$ to meet the user specification for a given process plant calls for real parameter optimization in fivedimensional hyperspace.

In order to solve the above optimization problem we have employed modified version of ABC namely L-ABC. This modified version was suggested by Rajasekhar et al. [14] and gave good performance in solving standard benchmark problems for different dimensions. Artificial Bee Colony $(\mathrm{ABC})$ is one of the most recent, swarm intelligence based algorithms employing the foraging behavior of honey bees for solving dynamic numerical optimization problems. ABC was suggested by Karaboga and Basturk for function optimization $[15,16]$ in 2005. Due to its simplicity and robustness it has been successfully applied to various practical optimization problems like Clustering [17]; IIR filter design [18]; extraction of MESFET [19] and so on; As pointed by Zhu and Kwong [20], the structure of ABC is such that it supports global exploration more in comparison to the exploitation. However, the performance of an 
algorithm depends on both exploration and exploitation phases for a required feasible solution. Various modifications have been suggested in literature to improve performance of $\mathrm{ABC}[21,22]$.Motivated by the performance of $\mathrm{L}-\mathrm{ABC}$ on various benchmark problems of higher dimension [14], in this present study we applied L-ABC to analyze the performance of FOPID controller in DC motor.

This paper is organized as follows: In section II, we present a mathematical model of DC motor as a controlled object. Section III gives a brief introduction of fractional calculus and its approximation. Section IV and V deals with Artificial Bee Colony (ABC) and proposed L-ABC respectively. In Section VI problem is formulated and Section VII presents the simulations and empirical results. Finally some conclusions are put forth in Section VIII.

\section{MODEL OF DC MOTOR}

In this approach we had considered the general model of DC motor which is shown in Fig 1. The applied voltage $V_{a}$ controls the angular velocity $\omega(t)$. The block model representation for the armature controlled DC motor is shown schematically in Fig 2. Transfer function of the motor has the form

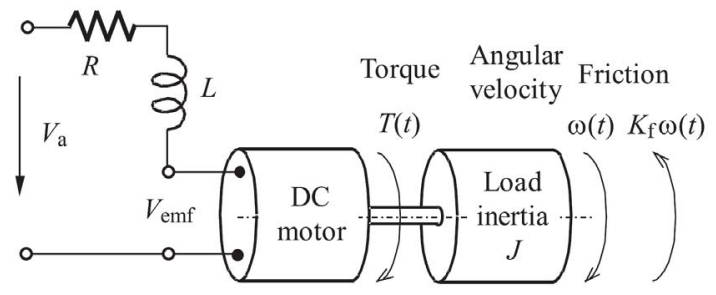

Fig 1. General Model of DC Motor

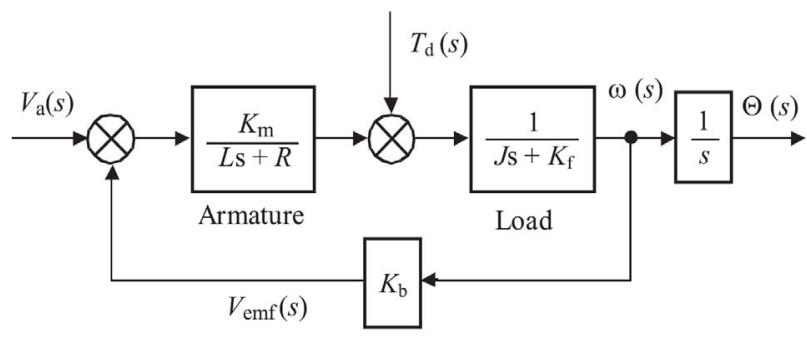

Fig 2. Mathematical Model of DC Motor

$$
G_{M}(s)=\frac{\theta(s)}{V_{a}(s)}=\frac{K_{m}}{s\left[(L s+R)\left(J s+K_{f}\right)+K_{b} K_{m}\right]}
$$

In many DC Motor the armature time constant is negligible and therefore a simplified model is being established and the continuous mathematical model has the following form [23]

$$
G_{M}(s)=\frac{\theta(s)}{V_{a}(s)}=\frac{K_{m}}{s\left[R\left(J s+K_{f}\right)+K_{b} K_{m}\right]}
$$

$$
=\frac{\left[K_{m} /\left(R K_{f}+K_{b} K_{m}\right)\right]}{s(\tau s+1)}=\frac{K_{M}}{s(\tau s+1)}
$$

Where the time constant $\tau=R J /\left(R K_{f}+K_{b} K_{m}\right)$ and $K_{M}=K_{m} /\left(R K_{f}+K_{b} K_{m}\right)$. In this armature controlled DC Motor it is interesting to note that $K_{m}=K_{b}$.

In this paper we had considered a mini DC motor with model number PPN13KA12C which is mainly used for robots, remote control applications, and many mechatronics applications as well. The following physical constants are taken in to consideration;

$R=6 \Omega$,

$K_{m}=K_{b}=0.1$,

$K_{f}=0.2 N-m s$

$J=0.01 \mathrm{Kg} \mathrm{m}^{2} / \mathrm{sec}^{2}$

By making use of above motor constants the transfer function has the form

$$
G_{M}(s)=\frac{0.08}{s(0.05 s+1)}
$$

In fig. 3 we depicted the bode diagram of DC motor (1) and we can observe that for given physical constant motor had good gain margin and phase margin as well.

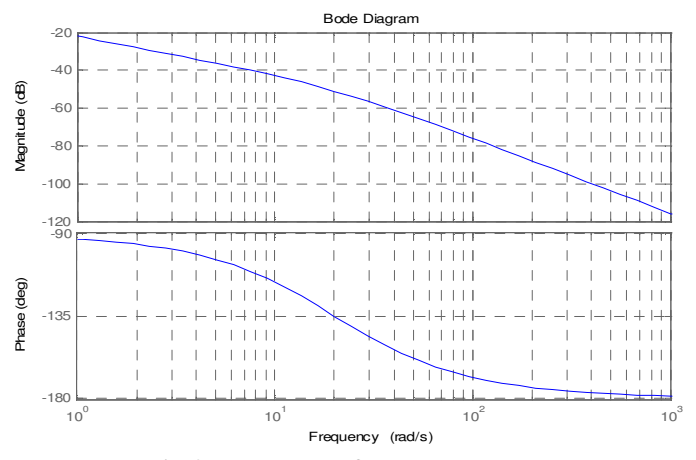

Fig 3. Bode plot of motor model

\section{FRACTIONAL ORDER SYSTEMS}

Fractional Calculus (FC) is the branch of mathematics having 300 years of history and which is gained the practical importance in recent years. FC is a generalization of ordinary differential calculus which considers the possibility of taking real number power of differential and integration operator. Generalized form of differ-integrator may be given as

$$
{ }_{a} D_{t}^{q} f(t)=\frac{d^{q} f(t)}{[d(t-a)]^{q}}
$$

where $q$ represents the real order of the differintegral, $t$ is the parameter for which the differintegral is taken and $a$ is the lower limit, in general considered to be 0 in almost all cases. Amongst all the definitions put forward for diiferintegral, Caputo's [24] definition for fractional derivative of order $\lambda$ with respect to variable $t$ is found to be 
most suitable in applications of fractional order controller design. The definition is as follows as

$$
\begin{gathered}
{ }_{0} D_{t}^{\lambda} y(t)=\frac{1}{\Gamma(1-\delta)} \int_{0}^{t} \frac{y^{(m+1)}(\tau) d \tau}{(t-\tau)^{\delta}} \\
(\gamma=m+\delta, m \in Z, 0<\delta \leq 1)
\end{gathered}
$$

where $\Gamma(Z)$ is Euler's gamma function. For $\gamma<0$, the formula for fractional integral of order $-\gamma$ is given as

$$
{ }_{0} D_{t}^{-\gamma} y(t)=\frac{1}{\Gamma(-\gamma)} \int_{0}^{t} \frac{y(\tau) d(\tau)}{(t-\tau)^{1+\gamma}} \quad(\gamma<0)
$$

Using Caputo's definition a fractional-order system may be defined by the following n-terms inhomogeneous fractional differential equation (FDE)

$$
a_{n} D^{\beta_{n}} y(t)+a_{n-1} D^{\beta_{n-1}} y(t)+\ldots .+a_{0} D^{B_{0}} y(t)=u(t)(5)
$$

Frequency domain transfer function of the system may be obtained through Laplace Transform of fractional derivatives as

$$
G_{n}(s)=\frac{1}{a_{n} s^{\beta_{n}}+a_{n-1} s^{\beta_{n-1}}+\ldots . a_{0} s^{\beta 0}}
$$

\section{ARTIFICIAL BEe COLONY AlgorithM}

Artificial Bee Colony $(\mathrm{ABC})$ algorithm classifies the foraging artificial bees into three groups; the employed bees, the onlooker bees and the scouts. The first half of the colony consists of the employed bees and second half consist of the onlooker bees. A bee that is currently searching for food or exploiting a food source is called an employed bee and a bee waiting in the hive for making decision to choose a food source is called an onlooker bee. For every food source, there is only one employed bee. The employed bee of abandoned food source becomes a Scout. In ABC algorithm each solution to the problem is considered as food source and is represented by a $D$-dimensional real-valued vector, where the fitness of the solution corresponds to the nectar amount of associated food source.

The algorithm starts by initializing all the employed bees with randomly generated food sources (solutions). In each generation/iteration every employed bee finds a food source in the neighborhood of its current food source and evaluates its nectar amount i.e., (fitness). In general the position of $i_{\text {th }}$ food source, for a D dimensional search space, is represented as $X_{i}=\left\{x_{i 1}, x_{i 2}, \ldots . x_{i D}\right\}$.After the information is shared by the employed bees; onlooker bees go to the region of food source at $X_{i}$ based on the probability $P_{i}$ defined as

$$
P_{i}=\frac{f i t_{i}}{\sum_{k=1}^{F S} f i t_{k}}
$$

$F S$ is total number of food sources. Fitness value $f i t_{i}$ is calculated by using following equation.

$$
\text { fit }_{i}=\frac{1}{1+f\left(X_{i}\right)}
$$

Here $f\left(X_{i}\right)$ is the objective function to be minimized. The onlooker finds its food source in the region $X_{i}$, by making use of following equation.

$$
x_{\text {new }}=x_{i j}+r *\left(x_{i j}-x_{k j}\right)
$$

Where $k \in(1,2,3, \ldots, F S)$ such that $k \notin i$ and $j \in(1,2,3, \ldots, D)$ are randomly chosen indexes, $r$ is a uniformly distributed random number in the range $[-1,1]$. If the obtained new fitness value is better than the fitness value achieved so far, than the bee moves to this new food source leaving this old one otherwise it retains the old food source. When all employed bees have completed this process, the information is shared with onlookers. Each of the onlookers selects a food source according to the probability given above. By this scheme good sources are well accommodated with onlookers. Each bee will search for a better food source for a certain number of cycles (limit), and if the fitness value doesn't improve then that particular bee becomes a Scout bee. A food source is initialized to that scout bee randomly and the search process continues. In this approach we used basic version which involve only one scout bee.

\section{L-ABC: LEVy MutATEd ARTIFICIAL BeE COLONY ALGORITHM}

According to Karaboga, $\mathrm{ABC}$ has only one control parameter 'limit' apart from the common control parameters of the population-based algorithms such as population size or colony size $(N B)$ and maximum generation number or maximum cycle number $(M C N)$. The basic version of $\mathrm{ABC}$ is very powerful in solving Uni-modal and Multimodal functions of considerable dimensions. However, the convergence rate and quality of solutions deteriorates with the increase in the problem complexity.

As mentioned earlier the basic structure of $\mathrm{ABC}$ favors exploration more in comparison to exploitation as a result some important domain knowledge may get lost during the successive iterations. In order to enhance the exploitation capabilities of $\mathrm{ABC}$, some added mechanism is needed for which we employed Levy mutation (based on Levy probability distribution) to assist $\mathrm{ABC}$ in exploitation of the search space.

\section{A. Levy Probability Distribution}

In 1930s, P. Levy, introduced a distribution which gives an infinite second moment, unlike finite second moment in Gaussian distribution, known as Levy's Probability Distribution [25]. It is also a stable process with an infinite moment which provides a characteristic tail at the end. The distribution is given as follows. 


$$
L_{\alpha, \gamma}(y)=\frac{1}{\pi} \int_{0}^{\infty} e^{-\gamma q^{\alpha}} \cos (q y) d q
$$

From Eqn. (10), we see that the distribution is symmetric with respect to $y=0$ and has two parameters $\gamma$ and $\alpha . \gamma$ is the scaling factor satisfying $\gamma>0$ and $\alpha$ should satisfy $0<\alpha<2$. Considering the limits in particular for $\alpha=1$, the integration can be analytically performed where it is equivalent to Cauchy Probability distributions. For limit $\alpha \rightarrow 2$, the distribution move towards Gaussian distribution. The parameter $\alpha$ controls the shape of the distribution in such a way that one can obtain different shapes of probability distribution, especially in the tail region.

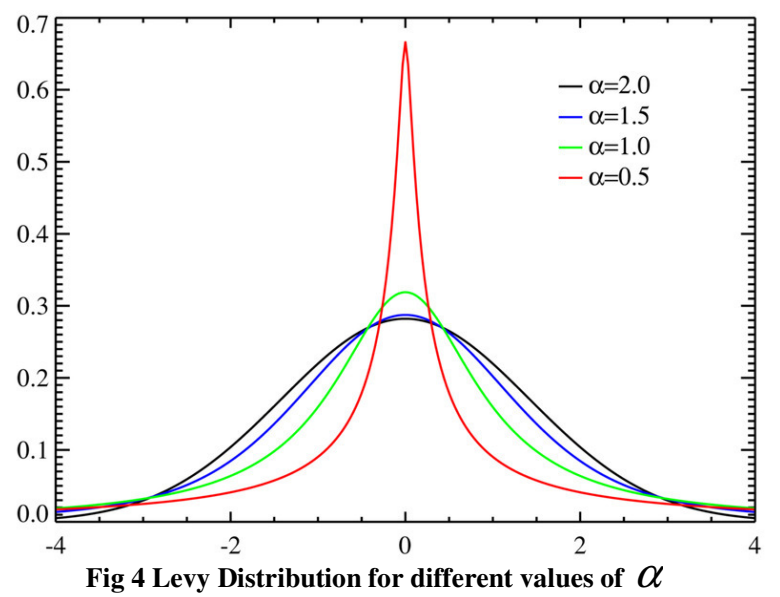

The smaller is the parameter $\alpha$ longer is the tail of the distribution. Levy distribution provides an ideal probability distribution for designing mutation operators because of its adjustable parameter. Different $\alpha$ values define probability distributions of various shapes. For example, it can implement both Gaussian and Cauchy distributions by simply changing the parameter $\alpha$. The complete mathematical and theoretical concepts of Levy Probability distribution can be found in [26].

\section{B. Levy Mutated Artificial Bee Colony}

The performance of Artificial Bee colony depends on exploration and exploitation which is done by the scout and employed bees respectively. The main motivation behind mutation is to guide the virtual bees towards the global optimum within less computational time (fast convergence) while maintaining the quality of solutions.

In order to apply Levy mutation we made use of Rechenberg's $1 / 5$ rule [22], which adapts the mutations step size depending on the frequency of successful mutations. This rule states that the ratio of successful mutations to all mutations should be $1 / 5$; hence if the ratio is greater than $1 / 5$ the step size increases, and if the ratio is less $1 / 5$ step size decreases. However, while performing the experiments we observed that the Rechenberg's ratio can be reduced further in case of $\mathrm{ABC}$ algorithm therefore in the present study we have taken the ration as $1 / 10$.

A new food source $X_{i}=\left\{x_{i 1}, x_{i 2}, x_{i 3}, \ldots, x_{i D}\right\}$ by L-ABC is generated as follows

$$
x_{\text {new }}=x_{i, b e s t}+L(\alpha, 0)
$$

Here $L(\alpha, 0)$ stands for random number generated by Levy's distribution. The mutation rule is applied making use of eq. (11).

\section{Pseudo code of ABC and Levy Mutated ABC}

Step1. Initialize the population of solutions $\quad x_{i j}, i=1,2, \ldots F S, \quad j=1,2, \ldots D$, trial $_{i}=0$ trail $_{i}$ is the non-improvement number

Step2. Evaluate the population

Step3. Cycle $=1$

Step4. REPEAT

\{----Produce new food source population for employed bee----- $\}$

Step5. For $i=1$ to $F S$ do

i. Produce a new food source $v_{i}$ for the employed bee of the food source $x_{i}$ by using (3) (in case of L-ABC use eq. (5) to evaluate the amount of nectar if Rechenberg's rule is not satisfied)

ii. Apply a greedy selection process between $v_{i}$ and $x_{i}$ and select the better one

iii. If solution $x_{i}$ doesn't improve trial $_{i}=$ trial $_{i}+1$, otherwise trial $_{i}=0$;

\section{End for}

Step6. Calculate the probability values $P_{i}$ by (4) for the solutions using fitness values.

$\{$---Produce new food source population for onlooker bee---

$$
\begin{array}{cl}
\text { i. } & t=0 ; \\
\text { ii. } & \mathrm{i}=1 ;
\end{array}
$$

\section{Step7. REPEAT}

If rand $<P_{i}$ then

i. Produce a new food source $v_{i}$ for the employed bee of the food source $x_{i}$ by using (3) (in case of L-ABC use eq. (5) to evaluate the amount of nectar if Rechenberg's rule is not satisfied)

ii. Apply a greedy selection process between $v_{i}$ and $x_{i}$ then select the better one

iii. If solution $x_{i}$ doesn't improve trial $_{i}=\operatorname{trial}_{i}+1$, otherwise trial $_{i}=0$;

iv. $\quad t=t+1$ 


\section{End if}

UNTIL $(t=F S)$

$\{-------D e t e r m i n e ~ S c o u t---------\}$

\section{Step8. If $\max ($ trial $)>$ limit then}

i. Replace $x_{i}$ with a new randomly produce solution by using following equation

$$
x_{i j}=x_{j}^{\min }+\operatorname{rand}(0,1) *\left(x_{j}^{\max }-x_{j}^{\min }\right)
$$

ii. Memorize the best solution achieved so far Cycle $=$ Cycle +1 ;

UNTIL (Cycle=Maximum Cycle Number)

\section{DESIGN OF FoPID CONTROLLER USING L-ABC}

A PID controller is a generic control feedback. In this process proportional control, integral control and derivative control trio are used together to minimize the error obtained between a measure process variable and a set value. Transfer function of an integer-order PID control may be given as

$$
G_{c}=K_{P}+\frac{K_{I}}{s}+K_{d} s
$$

However, incorporating fractional orders for integral and derivative control increase the controllability of the system and thus transfer function for fractional-order PID controller may be defined as

$$
G_{c}=K_{P}+K_{I} s^{-\lambda}+K_{d} s^{\mu}
$$

Here the parameters are $K_{P}, K_{I}, K_{d}, \mu$ and $\lambda$. There are various performance criteria for designing controller, of which some are integral absolute error (IAE), integral squared error (ISE). The main drawback of using these ISE and $I A E$ criteria is a dynamic response with a relatively less overshoot but a heavy settling time because they will calculate the errors uniformly over time [27]. If integral-time weighted squared error (ITSE) is used the drawback of prior mentioned methods can be eliminated, but it cannot ensure to have a desirable stability (and also it had more computational complexity). So, we made use of Integral-time-weightedabsolute-error (ITAE) which has an advantage of producing lesser oscillations and overshoot along with less settling time, which is mathematically defined as

$$
\text { ITAE }=\int_{0}^{\infty} t(|e(t)|)
$$

Our aim is to find out an optimal solution set $\left(K_{P}, K_{i}, K_{d}\right.$, $\mu, \lambda)$ for which ITAE $=0$. To find the appropriate set of parameters that leads to optimal value of ITAE, a five dimensional search space is selected for L-ABC; the dimensions being $\left(K_{P}, K_{i,} K_{d}, \mu, \lambda\right)$ and in case of L-ABC for the given five dimension the $i_{t h}$ food source is represented as it may be represented as

$$
X_{i}=\left[K_{p}, K_{I}, K_{d}, \lambda, \mu\right]
$$

Practical considerations of PID controller require that the parameter set adheres to the following constraints.

$$
1 \leq K_{P} \leq 5
$$

$$
\begin{aligned}
& 0 \leq \lambda, \mu \leq 1 \\
& 1 \leq K_{i}, K_{d} \leq 1
\end{aligned}
$$

Fig. 5. depicts the feedback control loop, where $\boldsymbol{C}(\boldsymbol{s})$ is the transfer function of controller and $\boldsymbol{G}(\boldsymbol{s})$ is the transfer function of the DC motor

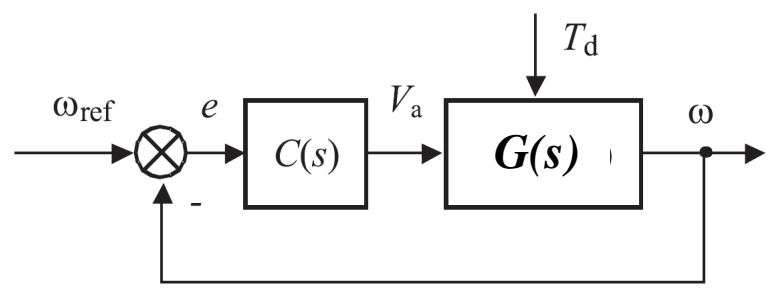

Fig 5 Feedback control loop of DC Motor

\section{EXPERIMENTAL RESULTS}

The proposed method has been tested on four specific instances of controller design for real life analog plant, which are summarized in table (1). For fractional-order differentiator $s^{r}$ is discretized using Tustin-based Continuous Fraction Expression (CFE) which may be given as

$$
D^{ \pm r}(z)=\left(\frac{2}{T}\right)^{ \pm r} \frac{P_{p}\left(z^{-1}\right)}{Q_{q}\left(z^{-1}\right)}
$$

Where, $T$ is the sampling period and, value of the expression for different degrees (i.e., different values of $p$ and q) are tabulated in Table 1 . In this paper we had considered sampling period to be $T=0.01 \mathrm{sec}$ and also we had made an approximation to be order as $p=q=5$.

Table 1 Expressions for Numerator and Denominator in CFE

\begin{tabular}{|c|l|}
\hline$p=q$ & $P_{p}\left(z^{-1}\right)(k=1)$ and $Q_{q}\left(z^{-1}\right)(k=0)$ \\
\hline 1 & $(-1)^{k} z^{-1} r+1$ \\
\hline 3 & $(-1)^{k}\left(r^{3}-4 r\right) z^{-3}+\left(6 r^{2}-9\right) z^{-2}+(-1)^{k} 15 z^{-1} r+15$ \\
\hline 5 & $\begin{array}{l}(-1)^{k}\left(r^{5}-20 r^{3}+64 r\right) z^{-5}+\left(-195 r^{2}+15 r^{4}+225\right) z^{-4} \\
+(-1)^{k}\left(105 r^{3}-735 r\right) z^{-3}+\left(420 r^{2}-1050\right) z^{-2} \\
\\
+(-1)^{k} 945 z^{-1} r+945\end{array}$ \\
\hline
\end{tabular}

Table 2 Algorithmic Parameters (ABC and L-ABC)

\begin{tabular}{|l|l|}
\hline Parameter & Value \\
\hline No of Bees (NB) & 20 \\
\hline Food Sources (FS) & $\mathrm{NB} / 2$ \\
\hline Employed bees & $50 \%$ of bees \\
\hline Onlooker bees & $50 \%$ of bees \\
\hline Scout bees & 1 \\
\hline Limit & $n_{e} * D$ \\
\hline Scale Parameter & 1.5 \\
\hline Generations & 100 \\
\hline
\end{tabular}




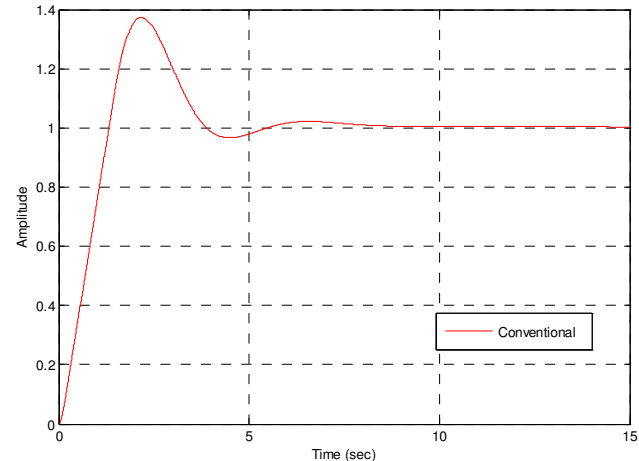

Fig 6 Unit Step Response of Conventional tuned FOPID Controller

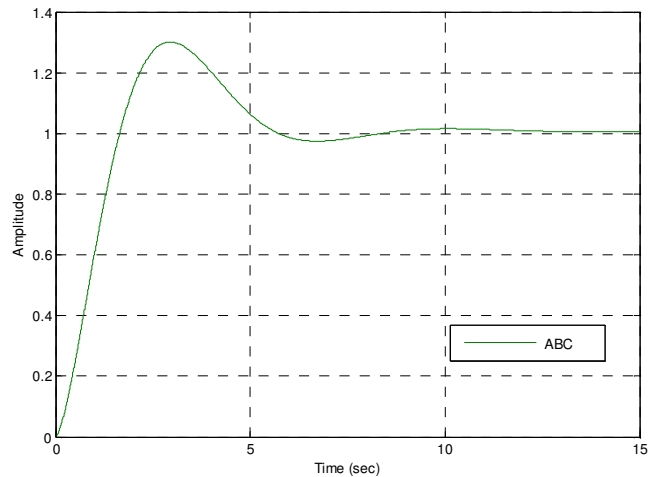

Fig 7 Unit Step response of ABC tuned FOPID Controller

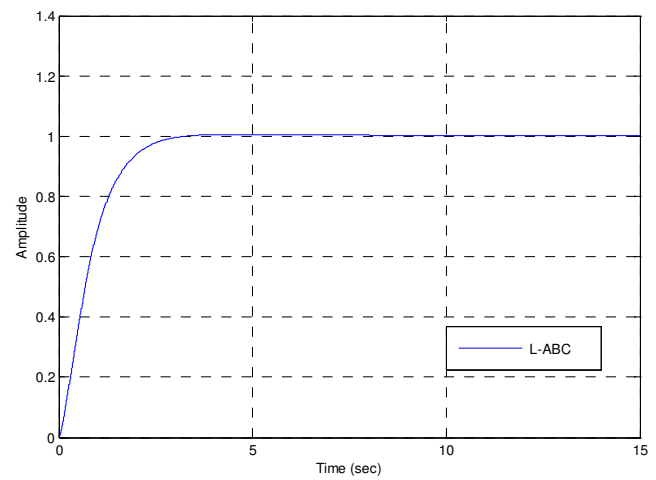

Fig 8 Unit Step response of L-ABC tuned FOPID Controller

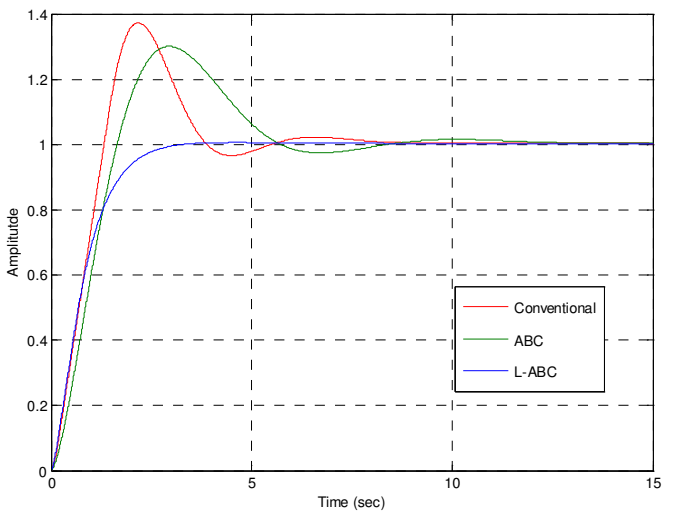

Fig 9 Comparison of Step responses of FOPID Controllers
Table 3 Comparison of Time Domain Indices for Proposed FOPID controller in a DC Motor

\begin{tabular}{|c|c|c|c|}
\hline $\begin{array}{c}\text { Method/ } \\
\text { Parameter }\end{array}$ & Conventional & ABC & L-ABC \\
\hline$K_{P}$ & 0 & 0.162 & 0.4969 \\
\hline$K_{I}$ & 10.3 & 12 & 15 \\
\hline$K_{D}$ & 0.514 & 0.616 & 0.9936 \\
\hline$\lambda$ & 0.4 & 0.212 & 0.0379 \\
\hline$\mu$ & 0.5 & 0.172 & 0.1707 \\
\hline Rise time (sec) & $\mathbf{1 . 0 0 2 3}$ & 1.1917 & 1.5461 \\
\hline Peak Overshoot & 39.7895 & 30.081 & $\mathbf{0 . 6 3 9 8}$ \\
\hline $\begin{array}{l}\text { Steady State } \\
\text { error }\end{array}$ & 0.101 & 0.0246 & $\mathbf{0 . 0 0 1 8}$ \\
\hline
\end{tabular}

\section{CONCLUSIONS}

In this paper a new modified version of $\mathrm{ABC}$ is used for designing fractional order PID controller for feedback control of DC motor. The superiority of proposed approach is demonstrated by comparing it with conventional and basic version of $\mathrm{ABC}$. Our future research will focus on applying these bees' algorithms in designing fractional order plants for Discontinuous models also.

\section{REFERENCES}

[1] K.B. Oldham and J. Spanier, The Fractional Calculus. Academic Press, New York 1974,

[2] C. H, Lubich, Discretized fractional calculus, SIAM Journal on Mathematical Analysis 17 (3): 704-719.

[3] A, Outsaloup, Fractional order sinusoidal oscillators: optimization and their use in highly linear FM modulators. IEEE Trans on Circuits and Systems 28(10), 1007-1009.

[4] Z. Chunna and Z. Xiangde, The application of fractional order PID controller to position servomechanism. Intelligent Control and Automation, 2008.

[5] I, Podlubny. Fractional-order systems and PI/sup/spl lambda//D/sup /mu//-controllers. Automatic Control, IEEE Transactions on 44(1):208-214.

[6] Y, Q, Chen., X, Xue., H, Dou. Fractional calculus and biomimetic control. In: Proceedings of the First IEEE International Conference on Robotics and Biomimetics (RoBio04), Shengyang, China, August, IEEE.

[7] Y, Q, Chen., H, Ahn., D, Xue, Robust controllability of interval fractional order linear time invariant systems. In: proceedings of the ASME 2005. International Desing Engineering Technical Conferencs \& Computers and Information in Engineering Conference, Paper \# DETC2005-84744, Long Beach, CA, September 24-28, pp. 1-9.

[8] M, Nakagawa and K, Sorimachi, Basic characteristics of a fractance device, IEICE Transactions on Fundamentals E75-A (12), 1814-1819.

[9] B.M, Vinagre., I Podlubny., L, Dorack., V Feliu. On fractional PID controllers; a frequency domain approach. In: Proceedings of IFAC Workshop on Digital Control-PID'00, Terrassa, Spain.

[10] I, Petras. The fractional order controllers: methods for their synthesis and application. Journal of Electrical Engineering 50(9-10), 284-288.

[11] L, Dorcak., I, Petras., I, Kostial., J, Terpak. State-space controller designfor the fractional-oder regulated system. In: Proceedings of the ICCC'2001, Krynica, pp. 15-20.

[12] Ma, Chengbin and Y, Hori. The application of fractional order PID controller for robust two-inertial speed control. In: Proceedings of the 
$4^{\text {th }}$ International Power Electronics and Motion Control Conference, Xi'an, August.

[13] K Astrom and T Hagglund. PID controllers:Theory, Design and Tuning. Instrument Society of America, Research Traingle Park.

[14] Anguluri Rajasekhar, Ajith Abraham and Millie Pant, Levy Mutated Artificial Bee Colonu Algorithm for Global Optimization, IEEE International Conference on Systems, Man and Cybernetics (IEEE SMC 2011), IEEE, Anchorage, USA, 2011.

[15] D. Karaboga and B. Basturk, A Powerful and Efficient Algorithm for Numerical Optimization: Artificial Bee Colony (ABC) Algorithm. Journal of Global Optim, 2007, 3(39):159-172,

[16] D. Karaboga and B. Basturk, On The Performance of Artificial bee Colony (ABC) Algorithm. Applied Soft Comp, 2008, 8(1): 697-697.

[17] D. Karaboga and C. Ozturk. A Novel Clustering Approach: Artificial Bee Colony (ABC) Algorithm. App Soft Comp, 2011, 11(1): 652657.

[18] N. Karaboga, A new design method based on artificial bee colony algorithm for digital IIR filters, Journl of Fran Inst, 346(4):328-348

[19] Samrat Sabat, Siba K Udgata and Ajith Abraham, Artificial Bee Colony Algorithm for Small Signal Model Parameter Estimation of MESFET, Engineering Applications of Artificial Intelligence, 2010.

[20] G. Zhu, S. Kwong.: Gbest-guided artificial bee colony algorithm for numerical function optimization. Applied Mathematics and Computation. Volume 217, Issue 7, pp. 3166-3173, 2010.

[21] A, Abraham, R.K. Jatoth and A, Rajasekhar.: Hybrid Differential Artificial Bee Colony Algorithm, Journal of Computation and Thheoretical Nanoscience, USA, 2011.

[22] Bahriye Akay, Dervis Karaboga.: A modified Artificial Bee Colony algorithm for real-parameter optimization, Information Sciences, In Press, DOI: 10.1016/j.ins.2010.07.015

[23] Dorf, R.C-Bishop, R.H.: Modern Control Systems, Addison-Wesley, New York, 1990

[24] M, Caputo. Elastictia e Dissipacione. Zanichelli, Bologna.

[25] Chang-Yong Lee; Xin Yao;, "Evolutionary programming using mutations based on the Levy probability distritbuion, "Evolutionary Computation, IEEE Trans on. Vol. 8, no. 1, pp. 1-13, Feb 2004.

[26] P. Levy, Theorie de l'Addition des Veriables, Aleatories, Paris, France: Gauthier-Villars. .

[27] R. A Krohling, J, P, Rey, "Design of Optimal Disturbance Rejection PID controller Using Genetic Algorithms". IEEE Trans. On Evolutionary Comp, 2001, Vol 5, pp. 78-82. 